\section{Morphium beim schweren Asthmaanfall?}

Durch einfache Fragen kann man schnell aus dem Konzept gebracht werden. Ein eindrucksvolles Beispiel aus der Medizin ist die Frage, woran ein Asthmatiker verstorben ist bzw. welches Organversagen zuerst zu seinem Tode geführt hat. Viele werden antworten, dass infolge der Schwere der Obstruktion oder/und der Mucusplaques der Gasaustausch nicht mehr möglich gewesen sei. Die daraus resultierende Hypoxämie habe zum Tode geführt. In der Klinik findet man aber auch bei einem schweren Asthmaanfall unter Sauerstoffgabe oft noch eine befriedigende Sauerstoffsättigung von über $85 \%$, so dass die Sauerstofftransportkapazität bzw. der Sauerstoffgehalt noch weit oberhalb des Normbereiches liegt. Trotzdem ist der Patient in extremis und muss unter Umständen intubiert werden.

Die Aussage, die Hypoxämie sei für den Tod verantwortlich, ist natürlich letztendlich richtig, denn eine längerdauernde Anoxie des Gehirns führt immer zum Tod. Die Hypoxämie steht jedoch nicht in der Kausalkette an vorderster Stelle. Die Luftnot beim Asthmatiker wird immer durch ein drohendes Versagen der inspiratorischen Atemmuskulatur (Atempumpe) verursacht. Die hohe Belastung der Atempumpe entsteht durch die bronchiale Obstruktion, welche zu einer Druckbelastung der Atemmuskulatur führt. Es kommt zum raschen Abfall der Energiereserven im Muskel mit anaerober Glykolyse, Abbau des Glykogens und intrazelluläre Azidose. Eine Nachlieferung von Glukose oder Fetten via Blut reicht nicht aus, um den Energiebedarf zu decken.

Diese pathophysiologische Erkenntnis ist nicht neu und seit vielen Jahren in der Literatur auffindbar, jedoch nicht sehr verbreitet. Sie hat aber direkte therapeutische Konsequenzen: Natürlich ist der Schwerpunkt der Therapie des akuten Asthmaanfalls die antiobstruktive Behandlung mit der Inhalation von Betamimetika im Hochdosisbereich, der antientzündlichen Behandlung mit Steroiden, der Gabe von Theophyllin - das vermutlich doch an der Muskulatur angreift und ggf. der therapeutischen Bronchoskopie, um Sekretpfröpfe $\mathrm{zu}$ entfernen. Besonders in schweren Asthmaanfällen führen diese Maßnahmen zumindestens in den ersten Stunden nicht immer zu dem erwünschten Rückgang der Luftnot der Patienten. Mit schlechtem Gewissen werden deswegen häufig von erfahrenen Klinikern Sedativa gegeben, die erfahrungsgemäß gut wirken. Mit schlechtem Gewissen deswe-

Pneumologie 2000; 54: 220-221

(c) Georg Thieme Verlag Stuttgart · New York ISSN 0934-8387

\section{Köhler}

Krankenhaus Kloster Grafschaft, Zentrum für Pneumologie, Beatmungs- und Schlafmedizin, Schmallenberg

gen, weil durch Sedativa eine Hyperkapnie induziert werden kann, die gemeinhin als gefährliche Nebenwirkung gilt. Hier hat sich in den letzten Jahren jedoch ein entscheidender Wandel vollzogen, der im Wesentlichen aus den neuen pathophysiologischen Kenntnissen der nicht-invasiven Beatmung und der Entwöhnung von Langzeitbeatmeten genährt wird.

Die durch eine sedierende Medikation verursachte Hemmung des Atmungszentrums mit konsekutiver Hypoventilation und Hyperkapnie vermindert deutlich den Energiebedarf der Atempumpe. Das Atemminutenvolumen ist im Einzelfall direkt proportional zur Atemarbeit, so dass z.B. eine Reduktion des Atemminutenvolumens von 10 auf 51 pro Minute eine Halbierung der Atemarbeit bedeutet. Durch das Unterschreiten der Erschöpfungsschwelle kann das unter Umständen lebensbedrohliche Versagen der Atemmuskulatur bzw. die Intubation verhindert werden. Gerade Asthmatiker haben infolge ihrer nur im schweren Anfall auftretenden Belastung der Atempumpe - im Gegensatz zur chron. obstruktiven Bronchitis mit Dauerobstruktion - keine ausreichende Anpassung des Atmungsreglers an diese lebensbedrohliche Situation. Es besteht noch keine veränderte Empfindlichkeitsschwelle des Atemzentrums (im Sinne der „Downregulation“). Der Asthmatiker versucht daher die durch die Obstruktion entstehende Hypoxämie kompensatorisch, mitunter auch überschießend, durch eine Hyperventilation zu beantworten. Dieses verstärkt aber wie erwähnt die Belastung der Atemmuskulatur.

Unter klinischen Bedingungen mit kontinuierlicher Überwachung (Pulsoxymetrie genügt) ist es deswegen therapeutisch wünschenswert, die Atmung zu bremsen, um die Atempumpe zu entlasten. Dieses ist am besten steuerbar mit Morphium, dessen körpereigene Äquivalente (Endorphine) physiologischerweise zur Dämpfung der Atmung benutzt werden. Es ist immer wieder erstaunlich, wie rasch die Dyspnoe eines Patienten sich nach intravenöser Gabe von $5 \mathrm{mg}$ Morphium bessert, ohne dass die Sauerstoffsättigung (unter $\mathrm{O}_{2}$-Gabe) in den pathologischen Bereich fällt. Meistens erreicht der Patient damit auch nicht den hyperkapnischen Bereich. Häufig steigt das $\mathrm{pCO}_{2}$, das infolge der relativen Hyperventilation oft bei $25 \mathrm{mmHg}$ liegt, nur auf Werte um 35-40 mm Hg an. Selbst wenn eine Hyperkapnie induziert wird, so kann dies in Kauf genommen werden, solange die Sättigung über $85 \%$ und der pH über 7,15 bleiben. Erst wenn dieser Bereich - vor allen Dingen im Zeitverlauf - unterschritten wird, muss man intubieren. Die klinische Erfahrung mit zahlreichen Intensivpatienten aus unserem intensivmedizinischen Zentrum 
zeigt aber, dass im schweren lebensbedrohlichen Asthmaanfall durch die konservative Therapie unter Einbeziehung von Morphium die Intubation häufig vermieden werden kann. Durch die Suppression des Atmungszentrums mit konsekutiver Entlastung der Atemmuskulatur über die induzierte Hypoventilation, kommt es über die Reduktion des Ernergieverbrauchs zum Hinausschieben der Erschöpfungsschwelle bis die antiobstruktive Therapie greift und damit die Ursache für die Belastung der Atempume entfällt.

Natürlich ist diese Therapie nicht ohne Überwachung möglich, weswegen sie außerhalb des Krankenhauses nicht durchgeführt werden sollte. Im Krankenhaus ist sie aber für den pneumologisch ausgebildeten Intensivmediziner eine sichere und zuverlässige Methode zur Behandlung des schweren Asthmaanfalls.

Ich habe in diesem Standpunkt bewusst auf Literaturangaben verzichtet, um die hoffentlich induzierte Diskussion nicht zu belasten. Ich würde mich sehr freuen, wenn dieser Beitrag den Leser dazu auffordert, eigene Erfahrungen und Kritik beizusteuern.

\section{Dieter Köhler}

Krankenhaus Kloster Grafschaft

Zentrum für Pneumologie, Beatmungs- und Schlafmedizin 57392 Schmallenberg 